\title{
In Vivo Activation of Mitogen-Activated Protein Kinases in Rat Intestinal Neoplasia
}

\author{
LAURA L. LICATO,* TEMITOPE O. KEKU, ${ }^{\ddagger}$ JOHN I. WURZELMANN, ${ }^{\ddagger}$ SHARON C. MURRAY, ${ }^{\ddagger}$ \\ JOHN T. WOOSLEY, § ROBERT S. SANDLER, ${ }^{\ddagger} \|$ and DAVID A. BRENNER ${ }^{\ddagger,\|,\|}$

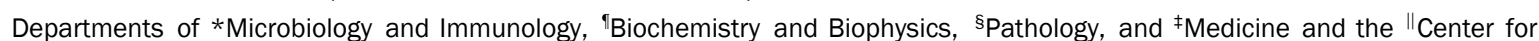 \\ Gastrointestinal Biology and Disease, University of North Carolina at Chapel Hill, Chapel Hill, North Carolina
}

Background \& Aims: To investigate whether mitogenactivated protein kinase (MAPK) cascades might play a role in the progression of colon cancer, c-Jun $\mathrm{N}$-terminal kinase (JNK) and extracellular signal regulating kinase (ERK) activity during colonic tumorigenesis were examined. Methods: The 1,2-dimethylhydrazine (DMH)-induced colon carcinoma model was used to study the activation of these kinases during intestinal carcinogenesis. Male Sprague-Dawley rats were injected with DMH for 24 weeks. Normal-appearing intestinal mucosa from control and treated animals and DMH-induced intestinal tumors were assayed for JNK and ERK activity using solid phase in vitro kinase assays. Tumors were typed for mutations in the K-ras gene. $\underline{R e}$ sults: There was little or no difference in JNK and ERK activity in hyperproliferative mucosa from DMH-treated animals compared with normal mucosa from control animals. However, in 16 colonic neoplasms, an average of 23-fold and 29-fold increases in JNK and ERK activities were observed, respectively, over control levels. In addition, activating protein-1 binding was strongly induced in the colonic tumors. Activation did not correlate with the presence of mutations in K-ras. Conclusions: Both the JNK and ERK MAPKs are highly activated during late progression of colorectal carcinoma. This change is dependent on the tumorigenic state rather than changes in proliferation.

$\mathrm{C}$ olonic carcinogenesis is a multistep process involving the accumulation of genetic mutations, including both the activation of oncogenes and the loss of tumor suppressor genes. ${ }^{1}$ Specific genetic alterations have been observed through the progression from a normal colonic epithelium to a hyperproliferative epithelium, adenoma, carcinoma, and invasive carcinoma. Patients with adenomas or carcinomas have a hyperproliferative epithelium compared with that of control subjects, even at sites distant from the adenoma or tumor. ${ }^{2-7} \mathrm{~A}$ field effect has been hypothesized, whereas global increases in intestinal proliferation at the cellular level increase the likelihood of new mutations, the accumulation of which leads to carcinogenesis. Alternatively, spontaneous mutations at the cellular level could enable individual cells to proliferate rapidly, leading to clonal expansion and an overall increase in intestinal proliferation. Unlike the later events in carcinogenesis in which specific mutations have been described, the molecular events that characterize intestinal hyperproliferation have not yet been identified.

Changes in proliferation can occur as a consequence of the activation of signal transduction pathways by mitogenic stimuli. Signal cascades that activate members of the mitogen-activated protein kinase (MAPK) family of proteins result in diverse cellular responses, including proliferation, differentiation, and neoplastic transformation. These changes are mediated by the activation of transcription factors, including the activating protein (AP)-1 transcription factor, which leads to changes in gene expression. AP-1 is implicated in both proliferation and tumorigenesis. ${ }^{8-10}$

Extracellular signals, such as cytokines or growth factors, stimulate signal cascades by binding to their specific receptors. ${ }^{11}$ This leads to activation of intermediate signaling molecules, such as the small guanosine triphosphate binding protein Ras, which transduce signals by activating a cascade of other serine/threonine kinases. Activation of these upstream S/T kinases leads to activation of dual-specificity kinases, such as MEK and SEK (JNKK), which then phosphorylate extracellular signal regulating kinase (ERK) and c-Jun $\mathrm{N}$-terminal kinase (JNK), respectively. Activated ERK and JNK translocate to the nucleus, where they directly activate transcription factors, including AP- $1 .{ }^{11}$ In addition to ras signaling pathways leading to activation of AP-1 through JNK and ERK proteins, the JNK pathway can also be stimulated by a ras-independent mechanism. ${ }^{12}$

\footnotetext{
Abbreviations used in this paper: AP, activating protein; $\mathrm{DMH}, 1,2-$ dimethylhydrazine; ERK, extracellular signal regulating kinase; JNK, c-Jun $\mathrm{N}$-terminal kinase; MAPK, mitogen-activated protein kinase; SDS, sodium dodecyl sulfate.

(C) 1997 by the American Gastroenterological Association 0016-5085/97/\$3.00
} 
The 1,2-dimethylhydrazine (DMH)-induced colon carcinoma model provides a reproducible system to study colonic carcinogenesis in vivo in rodents. Long-term treatment with DMH induces necrosis of colonic crypt cells, resulting in a compensatory proliferative response that leads to hyperplasia, dysplasia, and eventually tumorigenesis. ${ }^{13}$ Clinical signs in treated animals mimic those seen in human disease, including diarrhea, bloody stools, and weight loss. ${ }^{14-16}$ Similar to human colorectal cancers, about $40 \%-60 \%$ of intestinal tumors found in DMH-treated animals have a mutated K-ras gene. ${ }^{17,18}$ Despite recent investigations into the role of K-ras mutations in colorectal carcinoma, the physiological meaning of these changes remains poorly understood. Although it seems that mutation of K-ras can mediate the morphological changes and altered growth properties of transformed cells, ${ }^{19}$ it does not seem to be required for the tumorigenicity of these cells ${ }^{20}$ or for tumorigenesis in vivo.

In this study, the DMH-induced colon carcinoma model was used to investigate the activation of ERK and JNK MAPKs in vivo at the normal, hyperproliferative, adenoma, and carcinoma stages of tumorigenesis. In addition, we used a fasting/refeeding model as an independent method for examining ERK and JNK kinases in hyperproliferative intestinal mucosa in vivo. This report demonstrates strong activation of AP-1 and its regulating kinases in the later stages of colonic carcinogenesis. Furthermore, we show that this change does not occur during hyperproliferation, but only in adenomas and carcinomas.

\section{Materials and Methods}

\section{Carcinogen Treatment}

Male Sprague-Dawley rats initially weighing $\sim 240$ $\mathrm{g}$ (Harlan, Indianapolis, IN) were injected subcutaneously with either $25 \mathrm{mg} / \mathrm{kg}$ body wt of DMH (Sigma Chemical Co., St. Louis, MO) in $1 \mathrm{mmol} / \mathrm{L}$ ethylenediaminetetraacetic acid (EDTA) buffer, pH 6.5, or buffer alone at weekly intervals for 24 weeks. Body weight, general health, and the appearance of any signs of disease (bloody stools or diarrhea) were monitored throughout the course of treatment. The animals were killed 25 weeks after the onset of treatment, and normal-appearing tissue from the jejunum, cecum, and transverse colon was removed, as well as any tumors. Tissue was immediately flash frozen in liquid nitrogen or fixed in $10 \%$ buffered formalin.

\section{Fasting/Refeeding Treatment}

Male Sprague-Dawley rats weighing $\sim 220$ g were either fasted for 48 hours or fed a pelleted diet (Purina Rat Chow; Ralston-Purina Co., St. Louis, MO) ad libitum with water available in all cages. Fasted animals were either not refed ( 0 hour) or refed and killed at 1,4 , or 18 hours after refeeding. Control animals were fed ad libitum and killed at the 18-hour time point. Sample tissue from the jejunum, cecum, and transverse colon was immediately flash frozen in liquid nitrogen or fixed in $10 \%$ buffered formalin.

\section{Whole Cell Extracts}

Intestinal mucosa was separated from the underlying muscularis mucosa by scraping a glass slide along the bowel wall. The dissection technique was confirmed by light microscopy. Mucosal cells were lysed in Dignam C buffer ( $420 \mathrm{mmol} /$ $\mathrm{L} \mathrm{NaCl}, 1.5 \mathrm{mmol} / \mathrm{L} \mathrm{MgCl}_{2}, 20 \mathrm{mmol} / \mathrm{L}$ HEPES [pH 7.0], $0.2 \mathrm{mmol} / \mathrm{L}$ EDTA, $25 \%$ glycerol, and $0.5 \mathrm{mmol} / \mathrm{L}$ dithiothreitol $)^{21}$ with protease and phosphatase inhibitors $(0.5 \mathrm{mmol} / \mathrm{L}$ Pefabloc [Boehringer Mannheim Corp., Indianapolis, IN], 0.1 $\mathrm{mmol} / \mathrm{L}$ 4-nitrophenyl phosphate, $0.04 \mathrm{mmol} / \mathrm{L} \beta$-glycerophosphate, $0.05 \mathrm{mmol} / \mathrm{L} \mathrm{Na}_{3} \mathrm{VO}_{4}, 40 \mu \mathrm{g} / \mathrm{mL}$ bestatin, $2 \mu \mathrm{g} /$ $\mathrm{mL}$ aprotinin, $0.54 \mu \mathrm{g} / \mathrm{mL}$ leupeptin, and $0.7 \mu \mathrm{g} / \mathrm{mL}$ pepstatin A) using disposable homogenizers kept between $0-4^{\circ} \mathrm{C}$ at all times. Tumors were lysed directly by homogenation in Dignam $C$ buffer (plus inhibitors) after mincing with a razor blade. Protein concentration was determined using the Bio-Rad Protein Assay (Bio-Rad Laboratories, Hercules, CA).

\section{Ornithine Decarboxylase Activity Assay}

Ornithine decarboxylase activity was measured in the jejunum and transverse colon tissue samples of rats in the fasting and refeeding experiment by a modification of the standard method. ${ }^{22}$ Reactions containing $12 \mathrm{mmol} / \mathrm{L}$ dithiothreitol, $1.72 \mathrm{mmol} / \mathrm{L}$ DL-ornithine, and $0.5 \mu \mathrm{Ci}$ of $\mathrm{DL}-\left[{ }^{14} \mathrm{C}\right]-$ ornithine were incubated for 1 hour at $37^{\circ} \mathrm{C}$, and the $\mathrm{CO}_{2}$ released trapped on filter paper was soaked in $200 \mu \mathrm{L}$ benzethonium hydroxide. Reactions were stopped by the addition of $30 \%$ trichloroacetic acid and subsequently counted in a scintillation counter. A difluoromethylornithine-corrected blank was used for all calculations. The protein concentration of the homogenates was determined by the Lowry method. ${ }^{23}$ The ornithine decarboxylase activity was calculated by subtracting the quantity of $\mathrm{CO}_{2}$ released by nonspecific decarboxylation and expressed as picomoles $\mathrm{CO}_{2}$ released per milligrams of protein per hour.

\section{Mobility Shift Assay}

AP-1 DNA binding activity was determined using a radiolabeled double-stranded consensus AP-1 site (5' TAAAGCATGAGTCAGGACACCTC $3^{\prime}$ ) from the human collagenase gene as a probe in a reaction with $5 \mu \mathrm{g}$ of protein, as described previously. ${ }^{24}$ Supershift reactions included $1 \mu \mathrm{L}$ of anti-c-Jun, anti-c-Fos, or anti-JunB antibodies (Santa Cruz Biotechnology, Santa Cruz, CA). The use of whole cell extracts rather than nuclear extracts in the electrophoretic mobility shift assay experiment was necessitated by the tissue being flash frozen. However, both c-Fos and c-Jun have been previously shown to be localized in the nucleus, ${ }^{25}$ and the use of whole cell extracts to measure DNA binding activity has been well documented. ${ }^{26,27}$ 


\section{Kinase Assays}

JNK activity was assessed in intestinal mucosa samples using an in vitro kinase assay as described previously. ${ }^{28}$ Briefly, recombinant guanosine triphosphate-c-Jun protein was used as a substrate in a reaction containing $25 \mu \mathrm{g}$ of whole cell extracts (prepared from jejunum, transverse colon, or tumor tissue) and $\left[\gamma_{-}{ }^{32} \mathrm{P}\right]$ adenosine triphosphate (ATP). ERK activity was assessed similarly by an in vitro immune complex kinase assay. ${ }^{29}$ Immunoprecipitation was performed on whole cell extracts using an anti-ERK-2 antibody (Santa Cruz, C-14; cross-reactive with ERK-1) and protein A-Sepharose beads. Immunoprecipitates were then incubated in a kinase reaction including recombinant guanosine triphosphate-Elk protein (containing the $\mathrm{C}$-terminal portion of c-Elk) as a substrate and $\left[\gamma-{ }^{32} \mathrm{P}\right]$ ATP.

\section{Western Blot Analysis}

Western blot analysis was used to measure JNK-1 and ERK protein levels. Proteins from whole cell extracts were first fractionated by $12.5 \%$ sodium dodecyl sulfate-polyacrylamide gel electrophoresis (SDS-PAGE) and then transferred to polyvinylidene difluoride membranes (Immobilon-P; Millipore Corp., Bedford, MA). After blocking for nonspecific binding, the membranes were probed with either anti-JNK-1 (Pharmingen, San Diego, CA; 1:250), anti-ERK-2 (Santa Cruz, C$14 ; 1: 400$ ), or anti-active-ERK (Promega Corp., Madison, WI; 1:2000) antibodies followed by appropriate horseradish peroxidase-conjugated secondary antibodies (1:1000). Additional blots contained JNK-1 protein immunoprecipitated from whole cell extracts before electrophoresis. An enhanced chemiluminescence system was used for detection of the proteins (Amersham International plc, Buckinghamshire, England). Before immunoblotting, ponceau $\mathrm{S}$ staining was used to demonstrate equal protein loading.

\section{Genotyping for K-ras Mutations}

DNA was extracted from paraffin-embedded samples as described. ${ }^{30}$ A 116 -base pair DNA fragment including codons 12 and 13 of the K-ras gene was amplified using the polymerase chain reaction and oligonucleotides from exon 1 as primers (5' CCTGCTGAAAATGACTGAGTA 3' and 3' CCTACTTATACTAGGATGCT $5^{\prime}$ ). Five microliters of crude DNA extract was amplified in a $50-\mu \mathrm{L}$ reaction containing $0.15 \mu \mathrm{mol}$ of each primer and $0.625 \mathrm{U}$ of Taq polymerase using an automated DNA thermal cycler (Perkin-Elmer Cetus, Norwalk, CT) for 40 cycles of denaturation $\left(90^{\circ} \mathrm{C}, 20\right.$ seconds) and annealing $\left(50^{\circ} \mathrm{C}, 20\right.$ seconds). Extension occurred during the increase in temperature between the annealing and denaturation steps. Control reactions containing all reagents except DNA were performed to confirm the absence of contamination. Amplified DNA products were analyzed by gel electrophoresis on $5 \%$ polyacrylamide gels. Polymerase chain reaction products were subcloned into the $\mathrm{pCR}$ vector using the TA cloning kit (Invitrogen Corp., San Diego, CA) following the manufac- turer's instructions. Between 30 and 50 subclones from each tumor were then screened for K-ras mutations by oligonucleotide hybridization, performed as described previously. ${ }^{18}$

\section{Statistical Analysis}

For the DMH model, proliferation in controls was compared with that of treated animals using Wilcoxon's rank sum test. ${ }^{31}$ The same test was used to compare levels found in controls to average tumor levels in treated animals. The Wilcoxon's rank sum test is a nonparametric test that uses the ranks rather than the actual measures to test for a difference between two groups. No assumptions about the underlying distribution of the data are necessary. For comparisons of tumor to nontumorous tissue from the same animal, average proliferation was calculated when there was more than one tumor, and pairwise differences between tumor levels and levels in nontumorous tissues were obtained. The Wilcoxon's signed rank test ${ }^{31}$ was used to test the hypothesis that the median difference between tumor levels and nontumorous tissue (for the group of treated animals) was equal to zero.

\section{Results}

\section{DMH Induction of Hyperproliferation and Tumors in Rat Intestines}

Twelve male Sprague-Dawley rats were injected subcutaneously with $25 \mathrm{mg} / \mathrm{kg}$ body wt of DMH at weekly intervals for 24 weeks. Six control animals were injected only with buffer and monitored similarly. Twenty-five weeks after the onset of treatment, the animals were killed and the small and large intestine excised by incisions in the duodenum and rectum. A total of 20 tumors were found in 11 of the 12 treated animals (91.7\%), with 4 animals having 1 tumor (25\%), 5 animals having 2 tumors ( $41.7 \%$ ), and 2 animals having 3 tumors (16.7\%). Histological examination confirmed that most samples appearing abnormal on gross examination were adenomas or carcinomas (Table 1). These varied in regard to their histological stage, size, and location.

To determine the proliferative status of the normalappearing intestinal mucosa from the treated and control animals, whole crypt mitotic counts were done. Significant increases in the number of dividing cells were found in both the jejunum and transverse colon of DMH-treated animals compared with controls (Figure 1; $P=0.0011$ ).

\section{AP-1 Binding Is Induced in Neoplasia}

One of the downstream targets of MAPK signal pathways is the AP-1 transcription factor, consisting of Jun-Jun family homodimers or Jun-Fos family heterodimers. Because AP-1 is thought to play a role in proliferation and tumorigenesis, we investigated AP-1 activation by assaying AP-1 binding activity. Using the DNA mobility 
Table 1. Analysis of Intestinal Tumors in DMH-Treated Rats

\begin{tabular}{|c|c|c|c|c|}
\hline Tumor & Histology & Location & Size $(\mathrm{cm})$ & K-ras \\
\hline TO & Infiltratin carcinoma & Jejunum & 1.50 & $13 \mathrm{D}$ \\
\hline $\mathrm{T} 1$ & Invasive epithelial tumor & Jejunum & 1.00 & $13 \mathrm{~A}$ \\
\hline $\mathrm{T} 2$ & Epithelial atypia, invasive carcinoma, irregular glands & Transverse colon & 0.20 & WT \\
\hline T3 & Epithelial atypia, ulcerated carcinoma & Jejunum & 0.30 & $12 \mathrm{D}$ \\
\hline $\mathrm{T} 4$ & Epithelial atypia, intramucosal carcinoma & Transverse colon & 0.30 & $12 \mathrm{D}$ \\
\hline $\mathrm{T}^{\mathrm{a}}$ & Normal epithelium & Transverse colon & 0.50 & ND \\
\hline T6 & Minimal invasive epithelial carcinoma & Jejunum & 0.70 & WT \\
\hline $\mathrm{T} 7$ & Adenomatous polyp with multiple lumina & Transverse colon & 0.40 & $12 \mathrm{D}$ \\
\hline $\mathrm{T}^{\mathrm{a}}$ & Normal epithelium & Transverse colon & 0.40 & WT \\
\hline T9 & Invasive epithelial carcinoma & Jejunum & 0.50 & $12 \mathrm{D}$ \\
\hline T10 & Epithelial atypia, invasive carcinoma & Transverse colon & 0.40 & WT \\
\hline T11 & Epithelial atypia, invasive carcinoma & Transverse colon & 0.40 & WT \\
\hline $\mathrm{T} 12$ & Epithelial atypia, glandular epithelial invasive carcinoma & Duodenum & 0.20 & $12 \mathrm{D}$ \\
\hline T13 & Villous adenoma, carcinoma & Duodenum & 0.50 & WT \\
\hline T14 & Frankly invasive intramucosal carcinoma & Transverse colon & 0.70 & $12 \mathrm{D}$ \\
\hline $\mathrm{T} 15$ & Invasive epithelial carcinoma & Duodenum & 0.40 & WT \\
\hline $\mathrm{T} 16$ & Intramucosal carcinoma & Transverse colon & 1.00 & $12 \mathrm{D}$ \\
\hline $\mathrm{T} 17^{a}$ & Normal epithelium, lymphoid nodules & Jejunum & 0.50 & WT \\
\hline T18 & Adenomatous polyp & Transverse colon & 0.30 & WT \\
\hline $\mathrm{T} 19$ & Adenomatous polyp & Jejunum & 0.20 & $12 \mathrm{D}$ \\
\hline
\end{tabular}

ND, not done; WT, wild type (12G, 13G).

${ }^{a}$ Sample that was omitted from the tumor group based on histological characterization.

shift assay, there was no detectable AP-1 binding activity in either normal intestinal mucosa from control animals or hyperproliferative mucosa from DMH-treated animals (Figure 2). However, equal amounts of protein extracts from adenomas and carcinomas showed AP-1 binding (Figure 2). Supershift experiments using antibodies against AP-1 proteins confirm that c-Jun, c-Fos, and JunB are components of this AP-1 complex (data not shown).

\section{ERK and JNK Activity Is Induced in Intestinal Tumors}

Both ERK and JNK pathways can regulate AP1 expression and activation. ${ }^{11}$ ERK phosphorylates and

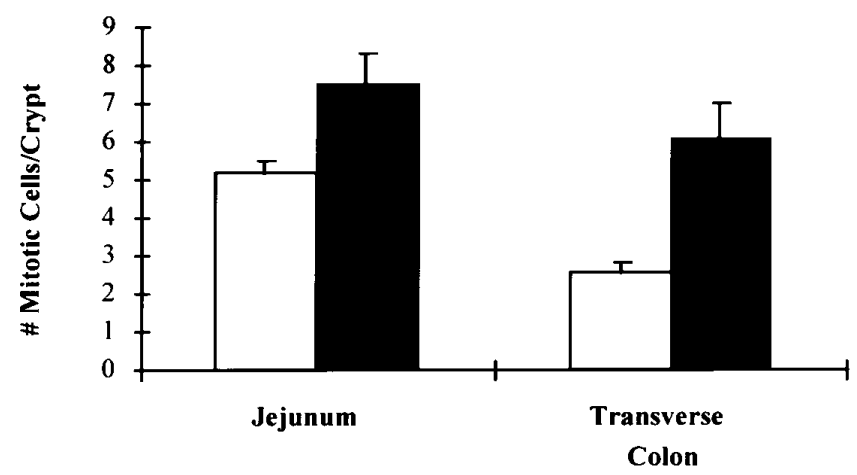

Figure 1. Intestinal mucosa from DMH-treated rats is hyperproliferative compared with mucosa from control animals. Whole crypt mitotic count analysis was performed on at least 20 dissected crypts per sample from the jejunum and transverse colon of DMH-treated and control $(\square)$ animals, as described in Materials and Methods.

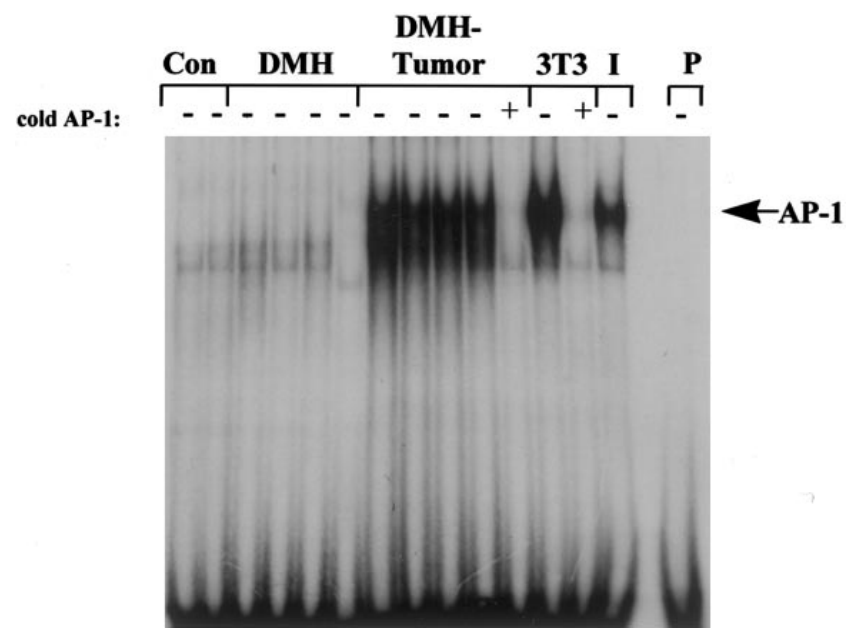

Figure 2. DMH-induced tumors show increased AP-1 binding activity. Whole cell extracts derived from transverse colonic mucosa in control (Con), DMH-treated (DMH), or intestinal tumors samples (DMH-Tumor) were analyzed for AP-1 binding activity using the DNA mobility shift assay. Binding reactions were performed by incubating $5 \mu \mathrm{g}$ of whole cell extract with a radiolabeled consensus AP-1 probe as described in Materials and Methods. DNA protein complexes were electrophoresed on a $5 \%$ nondenaturing polyacrylamide gel, followed by autoradiography. Some reactions contained a 500 -fold excess of unlabeled AP-1 probe as a competitor (+) to demonstrate specificity Additional competition reactions were performed with a similarly sized probe representing the consensus binding site of the Sp1 transcription factor (data not shown). Whole cell extracts from the serum-induced murine fibroblas NIH ЗT3 cell line (3T3) and the rat intestinal mucosal cell line IEC-6 (I) were used as positive controls. The last lane contains only radiolabeled AP-1 probe $(\mathrm{P})$. 
activates the Elk-1 transcription factor, which up-regulates expression of the c-fos gene by binding to a serum response element site in the c-fos promoter. JNK activates AP-1 by several mechanisms. First, JNK phosphorylates and activates the c-Jun protein directly, increasing AP1 activity. Second, because the c-jun gene contains an AP-1 site in its own promoter, activating c-Jun results in up-regulation of the expression of the c-jun gene. In addition, JNK can also phosphorylate the activation domain of Elk-1 and consequently upregulate c-fos expression. ${ }^{32}$ To determine which MAPKs are regulating AP-1 activation in intestinal neoplasias, ERK and JNK activities were measured using an in vitro kinase assay. As shown in Figure 3, there was a strong activation of JNK activity in intestinal neoplasias compared with intestinal mucosa from the transverse colon of control rats (23-fold average; $P=0.0024$ ) or compared with the hyperproliferative mucosa from the transverse colon of DMH-treated animals (8.4-fold average; $P=0.0078$ ). There was a small but significant increase $(2.7$-fold average; $P=0.0057)$ in JNK activity in hyperproliferative
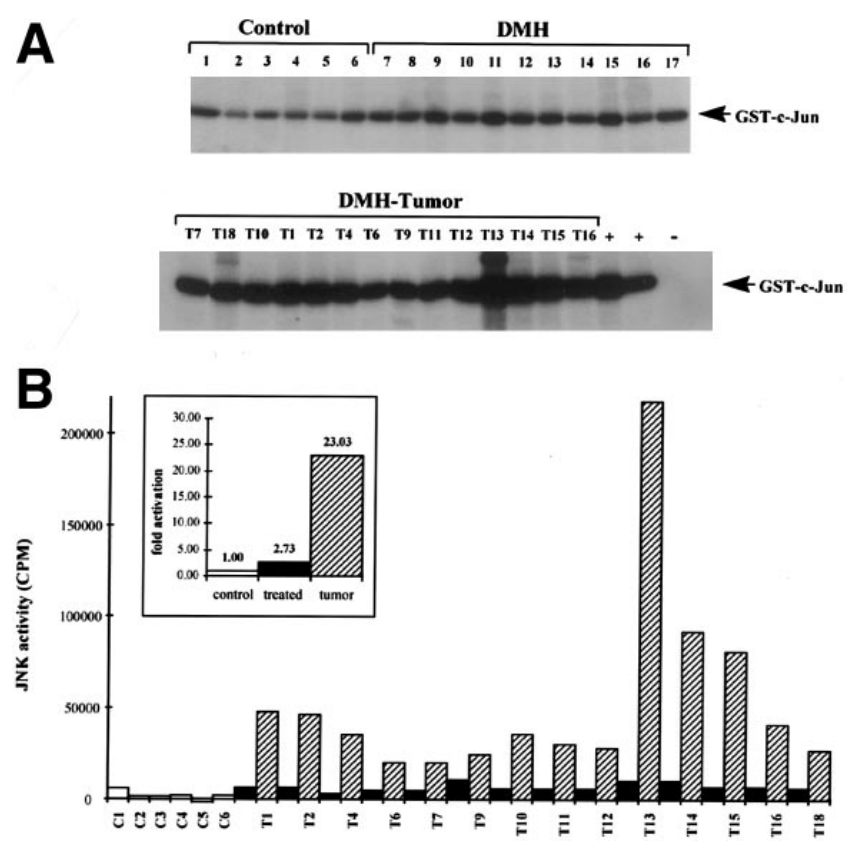

Figure 3. JNK activity is greatly increased in DMH-induced tumors. (A) Recombinant guanosine triphosphate-c-Jun protein was incubated with $50 \mu \mathrm{g}$ of whole cell extract from the transverse colon of control rats (Control, 1-6), DMH-treated rats (DMH, 7-17), or DMH-induced tumors (DMH-Tumor). Phosphorylation of guanosine triphosphate-cJun by extract-derived JNK was measured by incorporation of $[\gamma-$ ${ }^{32}$ PJATP as determined by SDS-PAGE and Phosphorlmager analysis. Extracts from $\mathrm{H}$-ras-activated and normal $3 \mathrm{~T} 3$ cells were used as positive controls $(+)$. Extract from normal $3 T 3$ cells, which was incubated with GST alone, was used as a negative control $(-)$. (B) Quantitation of activity shown in $A$ from control $(\square)$, DMH-treated ( $\square$ ), or tumor (跑) samples. Inset box shows group averages. transverse colon from DMH-treated animals compared with levels of JNK activity in transverse colon from controls. JNK activity was measured in intestinal mucosa from the jejunum of control and DMH-treated rats. Although increased JNK activity in hyperproliferative mucosa from treated animals was observed, the difference between control and DMH-treated animals did not reach significance. There were no significant regional differences in either JNK or ERK activity for intestinal mucosa or tumors.

ERK activity was more highly stimulated in intestinal tumors than JNK activity (Figure 4). Neoplastic tissue displayed an approximate 29-fold increase of ERK activity compared with normal-appearing mucosa from the transverse colon of controls $(P=0.0024)$ and a 23fold increase over normal-appearing mucosa from $\mathrm{DMH}-$ treated animals $(P=0.0078)$. There was no significant difference in ERK activity seen between hyperproliferative intestinal mucosa from DMH-treated animals and normal mucosa from control rats in either the transverse colon or jejunum.

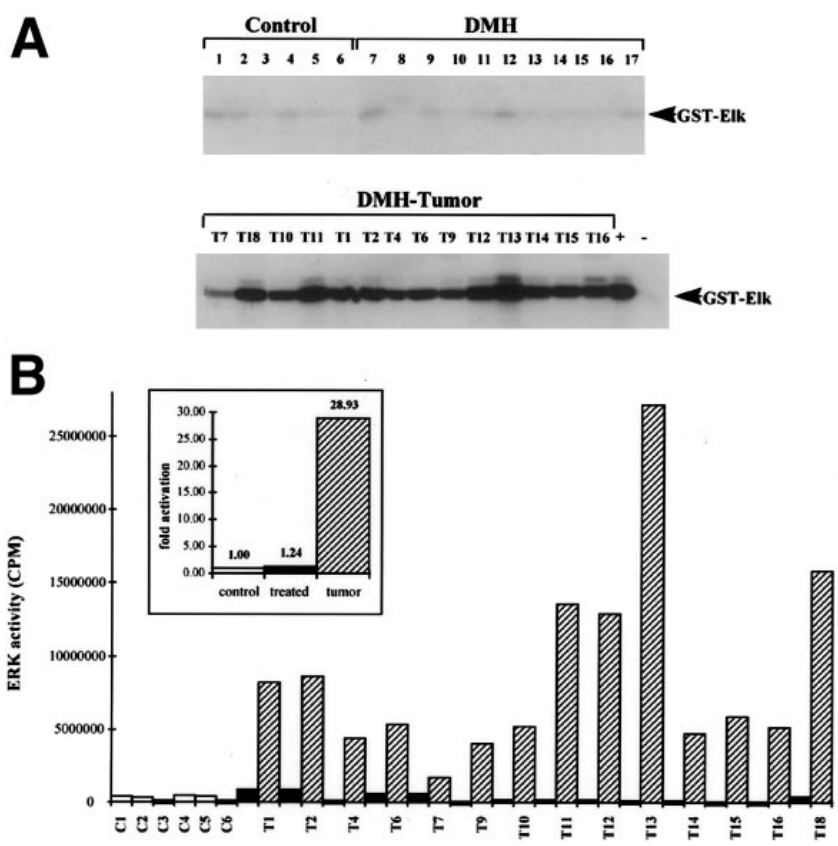

Figure 4. ERK activity is highly stimulated in DMH-induced tumors. (A) ERK-1 and ERK-2 proteins were immunoprecipitated from $50 \mu \mathrm{g}$ of whole cell extract from the transverse colon of control rats (control, 1-6), DMH-treated rats (DMH, 7-17), or DMH-induced tumors (DMHTumor) using a cross-reactive anti-ERK-2 polyclonal antibody. In vitro kinase reactions were performed on the immune complexes using recombinant guanosine triphosphate-Elk protein as a substrate and $\left[\gamma^{32}\right.$ P]ATP as a phosphodonor. Reactions were subjected to SDS-PAGE and Phosphorlmager analysis. Extracts from 3ТЗ cells were used as a positive control when incubated with guanosine triphosphate -Elk $(+)$ and as a negative control when incubated with GST alone $(-)$. (B) Quantitation of activity shown in $A$ from control ( $\square$ ), DMH-treated (ם), or tumor (沟) samples. Inset box shows group averages. 


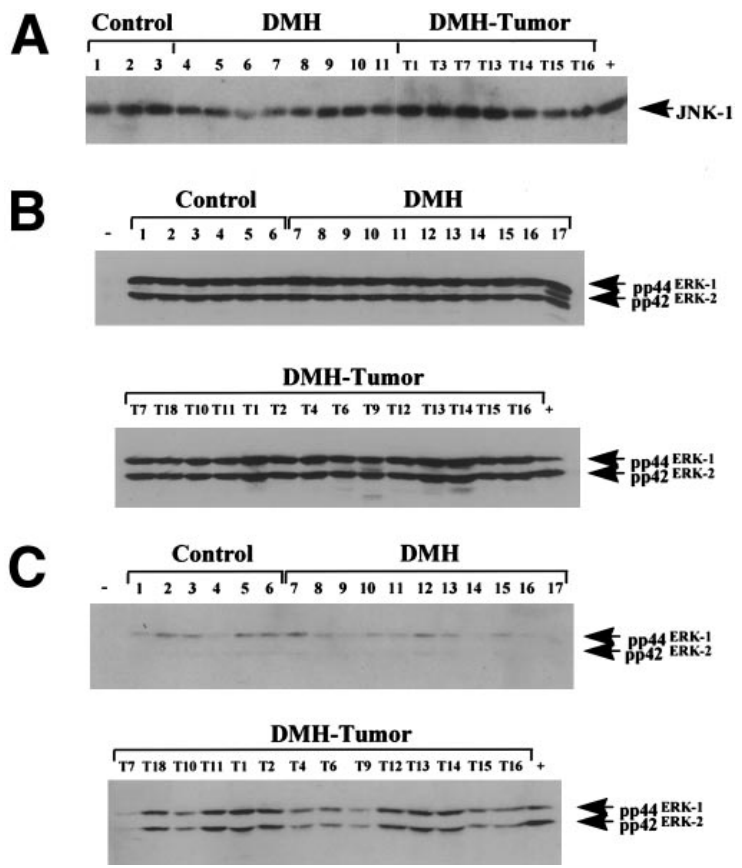

Figure 5. MAPK protein levels remain stable in DMH-induced tumors. Whole cell extract $(50 \mu \mathrm{g})$ from the transverse colon of control rats (Control), DMH-treated rats (DMH), or intestinal tumors (DMH-Tumor) was separated on $10 \%$ SDS-polyacrylamide gels and then transferred to immobilon-P membranes for Western blot analysis. Immunoblots were probed with $(A)$ a monoclonal anti-JNK-1 antibody, $(B)$ a polyclonal anti-ERK2 antibody, or $(C)$ a polyclonal anti-active ERK antibody and detected using chemiluminescence. Extract from 3T3 cells containing an oncogenic $\mathrm{H}$-ras gene (3T3-H61L) was used as a positive $(+)$ control.

To investigate whether any increased JNK and ERK kinase activity observed in DMH-induced neoplasia could be attributed to an increase in JNK or ERK protein levels, Western analysis was performed. No difference in JNK or ERK protein expression in DMH-induced tumors compared with mucosa in control animals or hyperproliferative mucosa from DMH-treated animals was observed (Figure $5 A$ and $B$ ). This suggests that the increase in JNK and ERK kinase activity observed is due to activation of these kinases by phosphorylation of their activating residues by upstream signaling kinases. We directly assessed this by reprobing the same Western blots with antisera specific for the phosphorylated form of the activation domain of ERK1/2. Figure $5 C$ shows an increased level of phosphorylated ERK in tumors compared with normal or DMH-treated mucosa.

\section{K-ras Mutations in Intestinal Tumors}

Because activating mutations in $\mathrm{K}$-ras occur in about $50 \%$ of colon cancers and Ras can activate both JNK and ERK, we examined the intestinal tumors induced by DMH treatment for K-ras mutations. Because tumor DNA represents a mixed population of cells, the following strategy was used to increase the sensitivity of detecting such mutations. Amplified K-ras products were subcloned and 30-50 subclones derived from each tumor were screened by DNA hybridization to wild-type and mutant K-ras oligonucleotides. A total of 9 of 16 tumors $(56 \%)$ were found to have mutations at the second nucleotide of either codon 12 or 13 in the K-ras gene (Table 1). Most of these were $G \rightarrow A$ transitions, as has been previously reported. However, one tumor contained a $G$ $\rightarrow \mathrm{C}$ transversion in codon 13 , a mutation that has been observed in human colon cancers. All mutations were confirmed by DNA sequencing (data not shown). When the K-ras genotype was compared with JNK and ERK activity, the presence of a K-ras mutation did not correlate with the kinase activity of either JNK or ERK.

\section{Intestinal ERK and JNK Are not Induced During the Hyperproliferative Response to Refeeding}

The above data suggest that the hyperproliferative state alone did not activate the ERK and JNK MAPKs. To separate the effects of intestinal hyperproliferation from those of tumorigenesis, we used a fasting/refeeding model to generate hyperproliferative intestinal mucosa within the context of normal intestinal growth and development. Male Sprague-Dawley rats were fasted for 48 hours and then refed for 1,4 , or 18 hours before they were killed. Control animals who were fed ad libitum were killed at the 18-hour time point. Samples of intestinal mucosa were then taken from the jejunum and transverse colon for analysis. Hyperproliferation in the refed intestinal mucosa was shown by measuring ornithine decarboxylase activity, whole crypt mitotic count, and ${ }^{3} \mathrm{H}$ thymidine incorporation assays (data not shown).

We examined intestinal mucosa in fasted and refed rats for JNK and ERK activity. There was no significant difference in the activity of these MAPKs, for either hypoproliferative or hyperproliferative tissue compared with tissue derived from ad libitum-fed control. This was true for mucosa from both the jejunum and the transverse colon (Figure $6 A-D$ ). Furthermore, there was no difference in the AP-1 binding activity of these samples using the mobility shift assay (data not shown). Western blot analysis shows similar amounts of JNK and ERK proteins in control, hypoproliferative, and hyperproliferative intestinal mucosa (Figure $6 E-H$ ). These observations suggest that hyperproliferation, whether induced by a carcinogen or fasting/refeeding, is not sufficient to activate the JNK and ERK MAPKs in intestinal mucosa in vivo. 


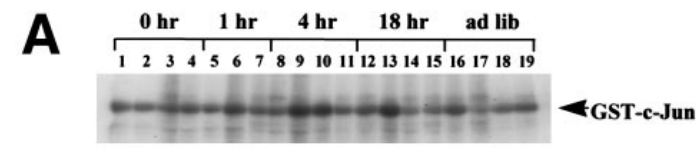

B

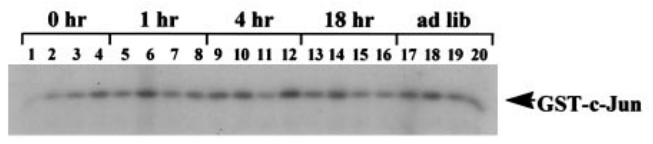

C
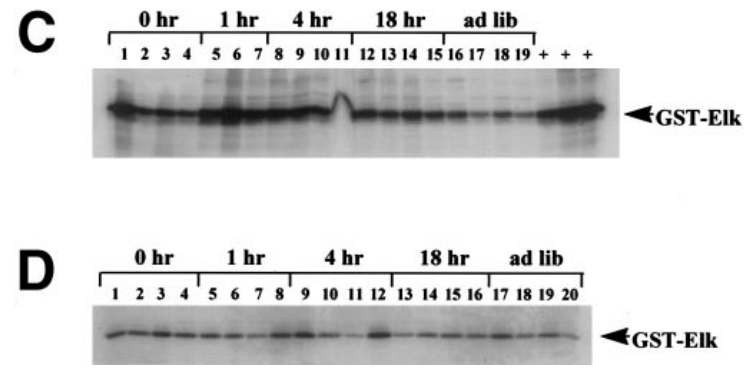

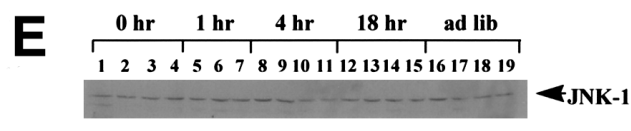

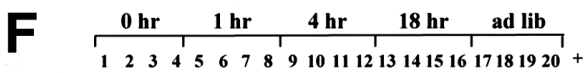
$12345567891011121314151617181920+$

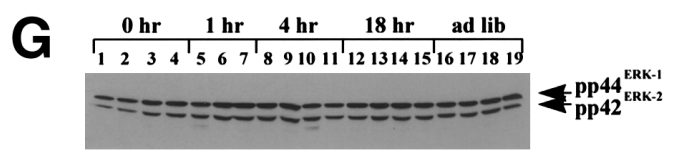

$\mathbf{H}$

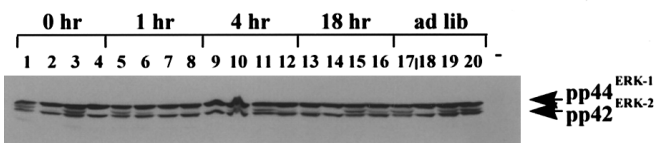

Figure 6. MAPK activity and protein levels do not change during fasting and refeeding treatment. $(A-D)$ Intestinal mucosa derived from the $(A)$ transverse colon or $(B)$ jejunum of fasted and refed rats was analyzed for JNK activity as described above. Extracts were from rats that were fasted for 48 hours and not refed ( $0 \mathrm{hr}$ ), refed for 1 hour (1 hr), 4 hours (4 hr), 18 hours (18 hr), or fed ad libitum throughout the experiment (ad lib). Similarly, ERK activity was assessed in extracts from the $(C)$ transverse colon or $(D)$ jejunum using the methods described. Positive controls $(+)$ included extracts from the serum-induced 3Т3 cell line and two separate extract preparations from 3T3 cells expressing an activated $\mathrm{H}$-ras gene, respectively. $(E-H)$ Western blot analysis was performed as described above using $50 \mu \mathrm{g}$ of whole cell mucosal extracts from the $(E$ and $G$ ) transverse colon or $(F$ and $H$ ) jejunum. Immunoblots were probed for $(E$ and $F)$ JNK-1 protein or $(G$ and $H)$ ERK proteins. Extracts from 3 T3 cells were used as positive $(+)$ and negative $(-)$ controls; the $(-)$ control lane was incubated with secondary antibody only.

\section{Discussion}

The early events leading to colorectal carcinogenesis are largely unknown. Although it has been observed that intestinal hyperproliferation precedes the formation of intestinal tumors, the relationship between hyperproliferation and the subsequent accumulation of mutations that mediate tumorigenesis is not clear. Because MAPK signaling plays a key role in proliferation, we have investigated the role of these pathways in colonic proliferation and tumorigenesis in vivo. This study demonstrates strong activation of both the JNK and ERK signal transduction pathways in intestinal adenomas and carcinomas, but not during intestinal hyperproliferation. Activation of JNK in adenomas and carcinomas was 23 -fold on average over that of control mucosa; ERK was activated on average 29-fold over control levels. In addition, AP1 binding activity was strongly induced in intestinal tumors. While both JNK and ERK regulate AP-1 activity, they do so via separate pathways and activation of different targets. ${ }^{11}$

These results contradict a previous study reporting a twofold decrease in activity of the ERK MAPKs in human colon tumors. ${ }^{33}$ This may reflect differences in ERK activity between humans and rats or may result from variation in the technical approaches. Increased levels of activated ERK protein in tumors was demonstrated in Western blots using antisera specific for the phosphorylated form of the ERK activation domain. However, Western blot analysis using antisera against total ERK protein showed similar amounts of ERK with no change in the apparent molecular weight between tumors and normal mucosa. This was a surprising result because previous studies in cultured cells showed a shift in the apparent molecular weight due to the phosphorylation of the ERK activation domain. However, this study and a recent study of primary gastric cells ${ }^{34}$ show this indirect assay for ERK activation is not valid in certain tissues. The absence of a shift in the apparent molecular weight may represent the net effect of multiple phosphorylation sites on ERK proteins.

Based on the different sizes, location, and histology of the adenomas/carcinomas induced by DMH treatment, different potential for growth and invasiveness would be expected between samples. Therefore, it is not surprising that the level of activation observed in JNK and ERK activity varied widely between tumors. It is interesting to note that the histological grade of 
adenoma/carcinoma did not predict the fold activation seen in the kinase activity, although we did observe a trend of increasing invasiveness with higher JNK or ERK kinase activity.

We did not observe activation of these kinases in hyperproliferative intestinal epithelium using two independent models, DMH treatment and fasting/refeeding. A hyperproliferative epithelium is thought to be the first histological change in the adenoma-carcinoma sequence of colonic carcinogenesis. ${ }^{1}$ However, the correlation between increases in proliferation and the risk of tumor development is controversial. Although a large body of data supports the idea that proliferative changes precede tumor formation, ${ }^{2,4,5,35,36}$ some studies fail to show a correlation between measurements of cellular proliferation and the risk of developing adenomas and carcinomas, ${ }^{37,38}$ calling into question the usefulness of proliferation measures as intermediate biomarkers. Our data show that activation of JNK and ERK, and consequent AP-1 activation, occurs at a stage after the proliferative changes seen in the intestinal mucosa during tumorigenesis. The fact that AP-1 activation correlates only with a change to frank neoplasia, and not with an increase in proliferation, suggests that AP-1 may play an important role in later neoplastic growth.

Both JNK and ERK pathways that regulate AP-1 can be activated by Ras. ${ }^{11}$ Mutations in K-ras occur in $40 \%-$ $50 \%$ of human colorectal carcinomas. ${ }^{39}$ Previous studies have shown that one third to two thirds of DMH-induced tumors have $\mathrm{K}$-ras mutations in the second position of codon 12 or 13 . Unlike K-ras mutations found in sporadic human colonic tumors, these mutations are almost exclusively G $\rightarrow$ A transitions. ${ }^{18}$ This is most likely due to the action of $\mathrm{DMH}$, which induces $O^{6}$-methylguanine adduct formation, thereby leading to $G \rightarrow A$ transitions as a result of mispairing. ${ }^{40,41}$ We show in the present study that 8 of $16 \mathrm{DMH}$-induced tumors have one of these K-ras mutations, while 1 additional tumor contains a $\mathrm{G} \rightarrow \mathrm{C}$ transversion. Although we cannot rule out other K-ras mutations undetectable with our probes, for example in codon 59, these are unlikely given previous studies in the DMH model. ${ }^{18}$

Because $\mathrm{K}$-ras mutations have been detected in both adenomas and normal mucosa adjacent to neoplasia, our results suggest that elevation of JNK and ERK activities occurs at a stage following K-ras mutation. Because both JNK and ERK can be activated through Ras, it is possible that this activation is a direct consequence of the $\mathrm{K}$-ras mutation. However, the level of JNK and ERK activation in individual DMH-induced tumors did not reflect the presence of a K-ras mutation. Furthermore, whereas all the adenomas and carcinomas examined in our study displayed elevated JNK and ERK activity, only $56 \%$ contained a mutation in K-ras. Thus, although activated Ras probably contributes to JNK and ERK activation in K-ras mutated tumors, it is unlikely that Ras pathways are the only mechanism responsible for the changes in activity of these MAPKs in colonic adenomas and carcinomas. Although in vitro studies using homologous recombination to knock-out $\mathrm{K}$-ras demonstrate a critical role for Ras in the growth characteristics of colon carcinoma cells, ${ }^{19,20}$ our data suggest that Ras effector pathways other than those leading to JNK and ERK activation may be most important.

Several known pathways may be responsible for the activation of JNK and ERK during colonic carcinogenesis. JNK can be activated by a TNF- $\alpha$-mediated Rasindependent pathway, whereas protein kinase $\mathrm{C}$ can activate the ERK pathway through direct activation of Raf- $1,{ }^{42-44}$ bypassing Ras. Furthermore, the elevated levels and activity of c-Src found during the progression of colonic carcinogenesis ${ }^{45-47}$ may contribute to the activation of JNK and ERK because v-Src has been shown to directly activate c-Raf, as well as cooperate with $\operatorname{Ras}^{48}$ and activate $\mathrm{JNK}{ }^{49}$

This study suggests that AP-1 activation may be important in colorectal cancers induced by the carcinogen DMH. This activation occurs by a complex mechanism, using at least two separate signal transduction pathways. It remains to be investigated whether similar results will be found in other genetic models, such as min mice, or naturally occurring colon cancers. A logical extension of this study is to determine if activation of AP-1 is necessary for colonic tumorigenesis. The DMH-induced colon carcinoma model can be used to address this question in vivo. The well-characterized nature of these signaling pathways will allow for individual components of these cascades to be manipulated and permit one pathway or the other to be selectively blocked. If blocking AP-1 activation prevents transformation, it may be possible to use inhibitors of this activation as therapeutic tools in human colorectal cancer. Finally, determining which AP1 responsive genes are important in tumorigenesis would allow for the identification of proteins that might be targeted to develop cancer therapies.

\section{References}

1. Vogelstein B, Kinzler KW. The multistep nature of cancer. Trends Genet 1993; 93:138-141.

2. Deschner EE, Maskens AP. Significanc of the labeling index and labeling distribution as kinetic parameters in colorectal mucosa of cancer patients and DMH-treated animals. Cancer 1982; 50: $1136-1141$. 
3. O'Brien MJ, O'Keane JC, Zauber A, Gottlieb LS, Winawer SJ. Precursors of colorectal carcinoma: biopsy and biologic markers. Cancer 1992; 70:1317 -1327.

4. Terpstra OT, van Blankenstein M, Dees J, Eilers GA. Abnormal pattern of cell proliferation in the entire colonic mucosa of patients with colon adenoma or cancer. Gastroenterology 1987; 92:704 -708.

5. Risio M, Lipkin M, Candelaresi G, Bertone A, Coverlizza S, Rossini FP. Correlations between rectal mucosa cell proliferation and the clinical and pathological features of nonfamilial neoplasia of the large intestine. Cancer Res 1991; 51:1917 -1921.

6. Lipkin M, Blattner WE, Fraumeni JF Jr, Lynch HT, Deschner E, Winawer S. Tritiated thymidine (phi p, phi h) labeling distribution as a marker for hereditary predisposition to colon cancer. Cancer Res 1983; 43:1899-1904.

7. Maskens AP, Deschner EE. Tritiated thymidine incorporation into epithelial cells of normal-appearing colorectal mucosa of cancer patients. J Natl Cancer Inst 1977; 58:1221 -1224.

8. Smeal T, Binetruy B, Mercola D, Grover-Bardwick A, Heidecker G, Rapp UR, Karin M. Oncoprotein-mediated signalling cascade stimulates c-Jun activity by phosphorylation of serines 63 and 73. Mol Cell Biol 1992; 12:3507 -3513.

9. Holt JT, Gopal TV, Moulton AD, Nienhuis AW. Inducible production of c-fos antisense RNA inhibits 3T3 cell proliferation. Proc Natl Acad Sci USA 1986; 86:4794 -4798.

10. Kovary K, Bravo R. The jun and fos protein families are both required for cell cycle progression in fibroblasts Mol Cell Biol 1991; 11:4466 -4472.

11. Karin M. The regulation of AP-1 activity by mitogen-activated protein kinases. J Biol Chem 1995; 270:16483 -16486.

12. Minden A, Lin A, McMahon M, Lange-Carter C, Derijard B, Davis RJ, Johnson GL, Karin M. Differential activation of ERK and JNK mitogen-activated protein kinases by Raf-1 and MEKK. Science 1994; 266:1719 -1723.

13. Simanowski UA, Wright NA, Seitz HK. Mucosal cellular regeneration and colorectal carcinogenesis. In: Seitz HK, Simanowski UA, Wright NA, eds. Colorectal cancer: from pathogenesis to prevention? New York: Springer-Verlag, 1989:225 -236.

14. LaMont JT, O'Gorman TA. Experimental colon cancer. Gastroenterology 1978; 78:1157-1169.

15. Freeman HJ, Kim Y, Kim YS. Glycoprotein metabolism in normal proximal and distal rat colon and changes associated with 1,2dimethylhydrazine -induced colonic neoplasia. Cancer Res 1978; 78:3385 -3390.

16. Ward JM. Morphogenesis of chemically induced neoplasms of the colon and small intestine in rats. Lab Invest 1974; 74:505 513.

17. Llor X, Jacoby RF, Teng BB, Davidson NO, Sitrin MD, Brasitus TA. K-ras mutations in 1,2-dimethylhydrazine -induced colonic tumors: effects of supplemental dietary calcium and vitamin D deficiency Cancer Res 1991; 91:4305-4309.

18. Jacoby RF, Llor X, Teng BB, Davidson NO, Brasitus TA. Mutations in the K-ras oncogene induced by 1,2-dimethylhydrazine in preneoplastic and neoplastic rat colonic mucosa. J Clin Invest 1991; 91:624 -630.

19. Shirasawa S, Furuse M, Yokoyama N, Sasazuki T. Altered growth of human colon cancer cell lines disrupted at activated Ki-ras. Science 1993; 260:85-88.

20. Plattner R, Anderson MG, Sato KY, Fasching CL, Der CJ, Stanbridge EJ. Loss of oncogenic ras expression does not correlate with loss of tumorigenicity in human cells. Proc Natl Acad Sci USA 1996; 93:6665-6670.

21. Sugarbaker JP, Gunderson LL, Wittes RE. Colorectal cancer. In: DeVita VT, Hellman S, Rosenberg SA, eds. Cancer: principles and practices of oncology. Philadelphia, PA: Lippincott, 1997: $800-803$
22. Slotkin TA, Bartolome J. Ornithine decarboxylase: marker of neuroendocrine and neurotransmitter actions. Methods Enzymol 1983; 103:590 -603.

23. Lowry OH, Rosebrough NJ, Farr AL, Randall RJ. Protein measurement with the folin phenol reagent. J Biol Chem 1951; 193:265 275.

24. Hattori M, Tugores A, Westwick JK, Veloz L, Leffert HL, Karin M, Brenner DA. Activation of activating protein 1 during hepatic acute phase response. Am J Physiol 1993; 264:G95 -G103.

25. Angel $P$, Karin M. The role of Jun, Fos and the AP-1 complex in cell-proliferation and transformation. Biochim Biophys Acta 1991; 1072:129 -157.

26. Imagawa M, Chiu R, Karin M. Transcription factor AP-2 mediates induction by two different signal-transduction pathways: protein kinase C and cAMP. Cell 1987;51:251-260.

27. Dent CL, Latchman DS. The DNA mobility shift assay. In: Latchman DS, ed. Transcription factors: a practical approach. New York: Oxford University Press, 1993:1-26.

28. Westwick JK, Brenner DA. Methods for analyzing c-Jun kinase. Methods Enzymol 1995; 255:342 -359.

29. Marais R, Wynne J, Treisman R. The SRF accessory protein Elk1 contains a growth factor-regulated transcriptional activation domain. Cell 1993; 73:381-393.

30. Yamaguchi K, Shirakabe K, Shibuya H, Irie K, Oishi I, Ueno N, Taniguchi T, Nishida E, Matsumoto K. Identificatio of a member of the MAPK family as a potential mediator of TGF- $\beta$ signal transduction. Science 1995; 95:2008-2011.

31. Remington RD, Schork MA. Statistics with applications to the biological and health sciences. 2nd ed. Englewood Cliffs, NJ: Prentice-Hall, 1970.

32. Whitmarsh AJ, Shore P, Sharrocks AD, Davis RJ. Integration of MAP kinase signal transduction pathways at the serum response element. Science 1995; 269:403 -407.

33. Attar BM, Atten MJ, Holian O. MAPK activity is down-regulated in human colon adenocarcinoma: correlation with PKC activity. Anticancer Res 1996; 16:395 -399.

34. Nakamura K, Zhou CJ, Parente J, Chew CS. Parietal cell MAP kinases: multiple activation pathways. Am J Physiol 1996; 271 : G640 - G649.

35. Deschner EE. Cell proliferation and colonic neoplasia. Scand J Gastroenterol Suppl 1988; 151:94-97.

36. Deschner EE. Cell turnover and colon tumor development. Prev Med 1987; 16:580 -585.

37. Hardman WE, Cameron IL. Site specifi reduction of colon cancer incidence, without a concomitant reduction in cryptal cell proliferation, in 1,2-dimethylhydrazine treated rats by diets containing $10 \%$ pectin with $5 \%$ or $20 \%$ corn oil. Carcinogenesis 1995; 16 : $1425-1431$.

38. Love RR, Surawicz TS, Morrissey JF, Verma AK. Levels of colorectal ornithine decarboxylase activity in patients with colon cancer, a family history of nonpolyposis hereditary colorectal cancer, and adenomas. Cancer Epidemiol Biomarkers Prev 1992; 1: $195-198$

39. Vogelstein B, Fearon ER, Hamilton SR, Kern SE, Preisinger AC, Leppert M, Nakamura Y, White R, Smits AM, Bos JL. Genetic alterations during colorectal-tumor development. N Engl J Med 1988; 88:525 -532.

40. Rogers KJ, Pegg AE. Formation of $O^{6}$-methylguanine by alkylation of rat liver, colon, and kidney DNA following administration of 1,2-dimethylhydrazine. Cancer Res 1977; 77:4082 -4087.

41. Pegg AE. Methylation of the $O^{6}$ position of guanine in DNA is the most likely initiating event in carcinogenesis by methylating agents. Cancer Invest 1984; 84; 2:223 -231.

42. Carroll MP, May WS. Protein kinase C-mediated serine phosphorylation directly activates Raf-1 in murine hematopoietic cells. J Biol Chem 1994; 269:1249 -1256. 
43. Kolch W, Heidecker G, Kochs G, Hummel R, Vahidi H, Mischak H, Finkenzeller G, Marme D, Rapp UR. Protein kinase C alpha activates Raf-1 by direct phosphorylation. Nature 1993;364: $249-252$.

44. Sozeri O, Vollmer K, Liyanage M, Frith D, Kour G, Mark GE, 3d, Stabel S. Activation of the c-Raf protein kinase by protein kinase C phosphorylation. Oncogene 1992; 7:2259 -2262.

45. Chen J, Compton C, Cheng E, Fromowitz F, Viola MV. c-Ki-ras mutations in dysplastic field and cancers in ulcerative colitis. Gastroenterology 1992; 102:1983 -1987.

46. Cartwright CA, Kamps MP, Meisler Al, Pipas JM, Eckhart W. pp60 ${ }^{\text {c-src }}$ activation in human colon carcinoma. J Clin Invest 1989; 83:2025 - 2033.

47. Cartwright CA, Meisler Al, Eckhart W. Activation of the pp60 ${ }^{\text {c-src }}$ protein kinase is an early event in colonic carcinogenesis. Proc Natl Acad Sci USA 1990; 87:558 -562.

48. Williams NG, Roberts TM, Li P. Both p21ras and pp60v-src are required, but neither alone is sufficient to activate the Raf-1 kinase. Proc Natl Acad Sci USA 1992; 89:2922 -2926.

49. Xie W, Herschman HR. V-src induces prostaglandin synthase 2 gene expression by activation of the c-Jun $\mathrm{N}$-terminal kinase and the c-Jun transcription factor. J Biol Chem 1995; 270:27622 27628.

Received January 23, 1997. Accepted July 21, 1997.

Address requests for reprints to: David A. Brenner, M.D., CB 7038 , 142 Glaxo Building, University of North Carolina at Chapel Hill, Chapel Hill, North Carolina 27599. Fax: (919) 966-7468.

Supported by National Institutes of Health-National Cancer Institute grants 5 P01CA50528, R01 7GM41804, DK-34987.

The authors thank Wuhan Jiang for assistance in tissue sectioning. Dr. Wurzelmann's present address is: ClinTrials Research Inc., Research Triangle Park, North Carolina. 